\title{
Jahres-Inhalt, Vol. 13, 1990
}

Übersichtsarbeiten

*Cour, V. (Neuilly s/Seine, France), Langenbahn, H. (München)

Fotemustin, ein neues Nitroso-Harnstoff-Derivat.

Aktueller Stand der Entwicklung

Originalarbeiten

Busse, E.; Kornhuber, B. (Frankfurt)

Veränderungen der intrazellulären Konzentrationen der zyklischen Nukleotide während der Differenzierung von

Neuroblastomzellen

12

Übersichtsarbeiten

Sass, H.-M. (Bochum)

Ethische Überlegungen zur klinischen Prüfung in Phase I

Edler, L. (Heidelberg)

Statistische Erfordernisse für Phase-I-Studien

Jost, L. M. (Zürich/Schweiz)

Überdosierung von Melphalan (Alkeran $\left.{ }^{\circledR}\right)$ : Symptome und

Behandlung. Eine Übersicht

85

90

96

Unterburger, P. (München) et al.

Diabetes mellitus und Mammakarzinom. Eine retrospektive

Verlaufsstudie

Mentges, B. (Mainz) et al..

Zum Steroidhormonrezeptorenstatus kolorektaler Karzinome .

Laufende klinische Studien

*Heidemann, E. (Stuttgart) et al.

Randomisierte Studie zum Vergleich von Mitoxantron mit

Epirubicin und Doxorubicin jeweils in Kombination mit Cyclophosphamid bei metastasierenden Mammakarzinomen .... .

17

21

14

Originalarbeiten

Aulbert, E. (Berlin), Steffens, O. (Essen)

Ferritin im Serum - Ein «Tumormarker» bei malignen

Lymphomen? 
Anger, B.; Schmeiser, T.; Heimpel, A. (Ulm), Robertson, J.; Sokal, J. E. (Durham, North Carolina, USA), Ganser, A. (Frankfurt), Carbonell, F. (Valencia, Spanien) Evaluation von 196 Patienten mit chronischer myeloischer Leukämie anhand eines Standard-Prognose-Modells....

102

L09

*Köppler, H. (Marburg) et al.

Behandlung hochmaligner Non-Hodgkin-Lymphome mit sequentieller oder alternierender Chemotherapie: Ergebnisse einer randomisierten multizentrischen Therapiestudie ....

$1 / 2$

Ludwig, Ch. U.; Ludwig-Hagemann, R.; Obrist, R.;

Holdener, E.E.; Sutter-Melde, C;

Obrecht, J. P. (Basel, Schweiz)

Verbesserte Verträglichkeit von alpha-Interferon durch eine

kontinuierliche subkutane Infusion

117

*Höffken, K. (Essen) et al.

Rekombinanter humaner Granulozyten-Makrophagen koloniestimulierender Faktor und niedrig dosiertes Cytosin-Arabinosid in der Behandlung von Patienten mit myelodysplastischem

Syndrom. Eine Phase-II-Studie ...

*Maschmeyer, G. et al. (Studiengruppe)

Antimikrobielle Interventionsstrategie bei neutropenischen

Patienten mit Fieber. Ergebnisse einer Multicenter-Studie bei

1260 Patienten mit hochmalignen Hämoblastosen

*Peest et al. (Studiengruppe)

Melphalan und Prednison (MP) versus Vincristin, BCNU,

Adriamycin, Melphalan und Dexamethason (VBAMDex)

Therapie beim multiplen Myelom. Erste Ergebnisse einer

multizentrischen Studie

$\mathrm{X}$

38

Peters, K. M. (Aachen), Beuth, J.; Kluger, J.;

Ko, H. L.; Grundmann, G.; Pulverer, G. (Köln)

Praoperative Inimunstimulation mit Propionibacterium avidum

KP-40 bei Patienten mit Magenkarzinom: Eine prospektiv

randomisierte Studie 124

Stierer, M.; Spoula, H.; Rosen, H. R. (Wien, Österreich)

Mammakarzinom beim Mann - Eine retrospektive Analyse von

15 Fallen 128

Laufende klinische Studien

*Kuse, R,; Calavrezos, A.; Küchler, R.;

Heilmann, H. P. (Hamburg)

Hohe Dosisintensität durch COPB1 AM beim Morbus Hodgkin mit ungünstigen Risikofaktoren $\quad 132$ 
*Ludwig, H.; Fritz, E.; Kotzmann, H.; Gísslínger, H. (Wien)

Erythropoietin-Behandlung der tumorassoziierten Anämie bei

Patienten mit multiplem Myelom

46

Kurzmitteilungen

Verhees, S. (Mannheim) et al.

Behandlung des fortgeschrittenen Pankreaskarzinoms mit

5-Fluorouracil, 4-Epidoxorubicin und Mitomycin C (FEMII).

Aus der Chemotherapiegruppe gastrointestinaler Tumoren

(CGT) 50

Abstrakts vom Internationalen Symposium

der Phase I/II-Studiengruppe, November 1989 in Frankfurt/M. . 54

Kongreßberichte $\quad 57$

Konsensusbericht 63

Editorial 5

Informationen für die Klinik $\quad 66$

Buchbesprechungen $\quad 68$

AIO-Mitteilungen $\quad 71$

*Bergmann, L.; Weidmann, E.; Runne, U.;

Mitrou, P.S. (Frankfurt), Keilholz, U. (Heidelberg),

Bartsch, H.H. (Göttingen),

Franks, C. R. (Amsterdam/Niederlande)

Interleukin-2 in Kombination mit Interferon-Alpha in der

Therapie des fortgeschrittenen malignen Melanoms und

Nierenzellkarzinoms - Eine Phase-I/II-Studie

137

Kurzmitteilungen

Kretzschmar, A.; Drings, P. (Heidelberg)

Epirubicin wöchentlich in der Kombinationschemotherapie mit

Cyclophosphamid und Vincristin beim unbehandelten

kleinzelligen Bronchialkarzinom: Eine Phase-II-Studie 141

Karstens, J. H.; Andreopoulos, D.; Ammon, J. (Aachen)

Initiale Tumorgröße und lokale Kontrolle nach

Radiochemotherapíe beim Nichtkleinzelligen

Bronchialkarzinom Stadium III 144

480

Jahres-Inhalt 1990

Kongreßberichte

Hossfeld, D. K. (Hamburg), Schauder, P. (Göttíngen) DAKE-AIO-Symposium «Ernährung und

Tumorerkrankungen» 146

Öhl, S. (Wuppertal)

AlO-Symposium «Geriatrische Onkologie - Perspektiven in der

Behandlung und Prävention» 146

Herrmann, R. (Berlin) 
Symposium «ZNS-Metastasen des Mammakarzinoms»

Mitteilungen der Deutschen Gesellschaft fur Hämatologie und

Onkologie $\quad-. \quad 149$

AIO-Mitteilungen $\quad 150$

* Aktuelle Therapiestudien - Referate gehalten anläßlich der 34. Jahrestagung der Deutschen

Gesellschaft für Hämatologie und Onkologie, Hannover 1989

Übersichtsarbeiten

Wolf, M.; Havemann, K. (Marburg)

Alternierende Chemotherapie beim kleinzelligen

Bronchialkarzinom 157

Ludwig, W.-D.; Thiel, E.; Teichmann, J.V. (Berlin),

van Dongen, J.M. (Rotterdam), Hofmann, J.; Riehm, H.

(Hannover)

Möglichkeiten und Grenzen der immunologischen Marker-

analysen zum Nachweis von minimaler residualer Erkrankung

bei akuter lymphoblastischer Leukämie im Kindesalter . . .

166

Originalarbeiten

Kleeberg, U.R.; Reichel, L. (Hamburg), Wander, H.-E. Beyer, J.-H. (Göttingen), Essers, U.

(Aachen), Fiebig, H.H. (Freiburg), Salewski, E.; Greifenberg, B. (Marburg), Edler, L.

(Heidelberg) Phase-П-Studie zur Wirksamkeit von Pirarubicin bei Patientinnen mit

metastasiertem Mammakarzinom . .

175

Drings, P.; Günther, I.U.; Edler, L. (Heidelberg), Gatzemeíer, U. (Großhansdorf), Berdel, W. (München), Stahl, M. (Hannover), Salewski, E. (Marburg) Pirarubicin in der Behandlung des fortgeschrittenen, nicht kleinzelligen Bronchialkarzinoms. Eine Therapiestudie der Phase-I/IIStudiengruppe der Arbeitsgemeinschaft Internistische Onkologie der Deutschen

Krebsgesellschaft .

181

Gatzemeier,U. (Großhansdorf), Drings, P.; Edler, L.;

Tessen, H.W. (Heidelberg), Fíebig, H.H. (Freiburg),

Hinke, A. (Ratingen), Rieche, K. (Gelsenkirchen)

Chemotherapie mit Tauromustin beim fortgeschrittenen

nicht-kleinzelligen Bronchialkarzinom. Eine Therapiestudie der

Phase-II-Studiengruppe der Arbeitsgemeinschaft Internistische

Onkologie der Deutschen Krebsgesellschaft 186

Queißer, W. (Mannheim), Preusser, P. (Münster),

Mross, K.B. (Hamburg), Fritze, D.(Darmstadt), Rieche, K.

(Gelsenkirchen), Beyer, J.-H. (Göttingen), Achterrath, W.

(Neu-Isenburg), Edler, L. (Heidelberg)

Phase-H-Prüfung von Carboplatin beim fortgeschrittenen

Ösophaguskarzinom. Eine Therapiestudie der Phase-I/II-

Studiengruppe der Arbeitsgemeinschaft Internistische

Onkologie der Deutschen Krebsgesellschaft 190 
Rath, U.; Manegold, C; Edler, L.; Schlag, P. (Heidelberg), Flechtner, H.; Queißer, W.

(Mannheim), Selbach, J. (Duisburg), Hạ́ung, H. (Darmstadt), Kabelitz, K. (Homburg), Trux,

F.A. (Siegen)

194

Etoposid, Adriamycin und Cisplatin (EAP) Kombinationschemotherapie beim fortgeschrittenen Magenkarzinom. A Phase-II-Therapiestudie der «Chemotherapiegruppe Gastrointestinaler Tumoren» (CGT) .

198

Scherübl, H.; Raue, F.; Ziegler, R. (Heidelberg)

Kombinationstherapie von Adriamycin, Cisplatin und Vindesin

beim C-Zell-Karzinom der Schilddrüse

Gheuens, E.; de Bruijn, E.A. (Antwerpen),

Slee, P.H.Th.J. (Nieuwegein)

203

Bioverfiligbarkeit von Cyclophosphamid im CMF-Regime .

Holtkamp, W.; Reis, H.E. (Aachen), Thiery, J. (München),

Rauschecker, H.; Nagel, G.A. (Göttingen)

Erniedrigte Plasmazinkspiegel beim metastasierten

Mammakarzinom

207

Ammon, A.; Eiffert, H.; Alhusen, R.; Weber, M.; Rümelin, B.;

Groh, E.; Bartsch, H.; Marschner, N.; Nagel, G.A.; Krieger, G.

(Göttingen)

«Mucin-Like Carcinoma-Associated Antigen»: Sensitivität und

Spezifität beim metastasierten Mammakarzinom

210

Kasuistiken

Weidmann, B.; Hoffmann, W.; Seeber, S. (Leverkusen)

Alpha2-Interferon beim Nierenkarzinom:

Erfahrungen an sechs Patienten 217

Zeymer, U.; Neuhaus, K.L. (Kassel)

Akute Koronarthrombose und Myokardischämie nach

Chemotherapie bei Morbus Hodgkin 221

Informationen für die Klinik 227

AIO-Mitteilungen 231

Buchbesprechungen 225

Übersichtsarbeiten

Höffken, K. (Essen)

Therapeutische Aspekte des Mammakarzinoms mit

günstiger Prognose 237

Berdel, W.E. (München)

Ether Lipide und Derivate als experimentelle

Antitumor-Substanzen 245

Originalarbeiten

Kraft, A.; Arnold, H.; Bodemann, H.; Hinkelbein, W.;

Wannenmacher, M. (Freiburg), v. Bültzingslöwen, F. 
(Donaustauf), Zwingers, T. (München)

Die Rolle der Kombinationschemotherapie mit oder ohne thorakalex Bestrahlung beim lokal-begrenzten kleinzelligen

Bronchialkarzinom. Eine randomisierte Multicenter

Phase-HI-Studie 253

Eindner, H. (Ingolstadt), Willich, N.; Schubert-Fritschle, G. (München), Atzinger, A. (Passau)

Die postoperative adjuvante Ganzabdomenbestrahlung beim

Ovarialkarzinom 260

Osmers, R.; Völksen, M.; Rath, W.; Kuhn, W. (Göttingen)

Vaginosonographie als Screeningmethode zur Erkennung von

Adnextumoren in der Postmenopause? 268

Jahres-Inhalt 1990

481

Marschner, N.; Nagel, G.A.; Beyer, J.-H.; Adler, M.;

Ammon, A. (Göttingen)

High-Dose Epirubicin in Koinbination mit Cyclophosphamid in der Behandlung des metastasierten Mammakarzinom.

Ergebnisse einer Dosisfindungs- und Phase-II-Studie 272

Kleine, H.D.; Birnbach, A.; Exeriede G., Link, H.;

Poliwoda, H,; Freund, M. (Hannover)

Therapieergebnisse und Prognose der akuten myeloischen

Leukämie im Erwachsenenalter: Retrospektive Studie von

319 Patienten zwischen 1977-1987 280

Heckmayr, M.; Gatzerneier, U. (Großhansdorf)

Megestroiacetat in der Behandlung kachektischer Patienten mit fortgeschrittenem Bronchialkarzinom 285

Füger, K.; Blumensteín, M.; Sauer, H. (München)

Dialysierbarkeit von Zytostatika. Experimentelle

Untersuchungen in vitro 289

Fleer, E.A.M.; Kim, D.-J.; Nagel, G.A.; EM, H.; Unger, C.

(Göttingen)

Zytotoxische Aktivität von Lysophosphatidylcholinanaloga in

humanen lymphoblastären Raji Zellen

295

Busse, E., Kornhuber, B. (Frankfurt)

Änderung der Adenylcyclase während der Differenzierung von

Neuroblastomzellen 301

Türeci, O.; Lagoda, P.; L·singer, O.-G. (Homburg),

Fischer, H. (Heidelberg)

Charakterisierung menschlicher Glioblastom Zellinien und nach

ihrer Heterotransplantation auf Nacktmäuse durch DNA

Fingerprinting 305

Kurzmitteilungen

Schumer, J.; Klocker, J.; Tidstrand, J.; Rab, B.; Allmayer, H.

(Klagenfurt/Österreich) 
Cis-Platin, Vindesin und Hyaluronidase in Kombination mit simultaner Strahlentherapie bei fortgeschrittenen Tumoren im Hals- und Kopfbereich

310

Marschner, N.W.;Adler, M.;Nagel, G.A. (Göttingen), Jaenicke, F. (München), Albrecht, U. (Bad Oldesloe) Langzeitergebnisse der antiemetischen Effektivität des 5-HT3-Antagonisten Ondansetron

313

Kasuistiken

Heim, M.E.; Hastka, J.; Queißer, W. (Mannheim)

Metastasierendes C-Zellkarzinom mit Knochenmarkkarzinose

bei multipler endokriner Neoplasie (MEN) Typ Ha.

Diagnostische und therapeutische Probleme 317

Informationen für die Klíník 322

AIO-Mitteilungen

325

Sonderbände 312

Buchbesprechungen 320

Editorial

Obrecht, J. P. (Basel, Schweiz)

Perspektiven der klinischen Onkologie für die 90er Jahre .... 333

Übersichtsarbeiten

Heidemann, E. (Stuttgart)

Therapeutische Aspekte des fortgeschrittenen Hochrisiko-

Mammakarzinoms 338

Mross, K. B.; Langenbuch, T.; Hossfeld, D. K. (Hamburg),

Burk, K. (Freiburg)

Jodo-Doxorubicin, ein neues Anthrazyklin-Derivat. Aktueller

Stand der Entwicklung $\quad 346$

Originalarbeiten

Weh, H. J.; Walter, T. A.; Kremer, B.; Henne-Bruns, D.; Crone-Muenzebrock, L. S.; Hossfeld, D. K. (Hamburg), Garbrecht, M. (München)

Behandlung des metastasierten Magenkarzinoms mit einem

modifizierten FAMTX Chemotherapie-Protokoll

351

Schuster, D.,Ade, N.; Heim, M. E. (Heidelberg), Schneider, G.

(Augsburg)

Kombinationstherapie mit rekombinantem alpha-2A-Interferon

und Vinblastin beim fortgeschrittenen Nierenzellkarzinom.... 359

du Bois, A.; Meerpohl, H. G.; Wilhelm, C,; Pfleiderer, A,

(Freiburg), Quaas, L.; Barnickel, I. (Bad Oldesloe)

Therapierefraktäre Emesis unter Cisplatin. Prospektive Studie

mit dem Serotonin-Rezeptor Antagonist GR 38032F364

Serve, H.; Perker, M.; Ertl, A.; Reichold, M.; Fink, U.; Berdel,

W. E. (München)

Ondansetron (GR 38032F), ein kompetitiver 5-HT3-Rezeptor- 
Antagonist als Antiemetikum bei Zytostatika-induzierter

Übelkeit und Erbrechen. Eine offene, substanzorientierte

Phase-П/IП-Studie 369

Henkel, E. (Heidelberg)

Eine Untersuchung zur Bewertung des Krebsinformations-

dienstes Heidelberg durch den Benutzer 375

Zoedler, T.; Becker, H.; Ebener, C.; Röher, H. D. (Düsseldorf),

Braasch, D. (Marburg)

Perfusionshyperthermie der Leber bei Schweinen -

Darstellung eines Verfahrens zur präzisen Steuerung der

extremen Hyperthermie 381

Klinisch-pathologische Konferenz

Obrist, R. (Basel, Schweiz), Wegmann, W.; Kiss, D. (Liestal,

Schweiz)

Prostatakarzinom mit einer ungewöhnlichen Verlaufsform .... 388

Kongreßberichte

Link, H.; (Hannover)

Pneumocystis-carinii-Pneumonie bei Immunsuppression in der

Hämatologie, Onkologie und bei Organtransplantation 392

Deutsche Krebshilfe e.V. (Bonn)

Workshop «Psychoneuroimmunology in Relation to Cancer»

vom 3.-7. Juni 1990 in Tutzing 393

Informationen für die Klinik

Kaufmann, M.; Bastert, G. (Heidelberg), Jonat, W.; Maass, H.

(Hamburg), Eiermann, W. (München)

Brusterhaltende Operation und adjuvante Therapie beim

Mammakarzinom 394

Mitteilungen onkologischer Gesellschaften

AIO-Mitteilungen 397

Sonderbände 396

Buchbesprechungen 399

482

Jahres-Inhalt 1990

416

Übersichtsarbeiten

Leib-Mösch, C; Brack-Werner, R.; Salmons, B.; Schmidt, J.;

Strauss, P. G.; Hehlmann, R.; Erfle, V.

Die Bedeutuag von Retroviren in der Onkologie $\quad 405$

Bergmann, L.

Klinische Bedeutung von Interleukin-2 .

Laufende klinische Studien

Peest et al.

Melphalan und Prednison (MP) versus Vincristin, BCNU, Adriamycin, Melphalan und Dexamethason (VBAMDex)

Induktionschemotherapie und Interferon Erhaltungstherapie 
beim Plasmozytom. Laufende Ergebnisse einer

multizentrischen Studie

458

424

Freund, M.; Hanauske, A.-R.

Interferon alpha in der Therapie der Non-Hodgkin-Lymphome

Originalarbeiten

Lenz, H. J.; Brunner, T.; Dopfer, R.; Schlag, H.; Ehninger, G.

Kontinuierliche Infusion von natiirlichem Interleukin 2 (n IL-2)

zur Behandlung maligner Erkrankungen: Phase-I-Studie 429

Berdel, W. E.; Danhauser-Riedl, S.; Steinhauser, G.;

Rastetter, J.

Stimulation des klonalen Wachstums menschlicher kolorektaler

Tumorzellen unter dem Einfluß von IL-3 und GM-CSF.

Modulation von 5-FU Zytotoxizität unter GM-CSF 437

Heî́m, M. E.; Siegmund, R.; Illiger, H. J.; Klee, M.; Rieche, K.; Berdel, W. E.; Edler, L.

Tumornekrosefaktor bei fortgeschrittenen kolorektalen

Karzinomen: Eine Phase-II-Studie. Eine Studie der Phase-I/II-

Studiengruppe der Arbeitsgemeinschaft Internistische

Onkologie der Deutschen Krebsgesellschaft

414

Weh, H. J.; Zügel, M.; Wingberg, D.; Schwarz, R-,- Zornig, C; Dietel, M.; Hossfeld, D. K. Chemotherapie metastasierter Weichteilsarkome mit einer Kombination aus Adriamycin/DTIC oder Adriamycin/Ifosfamid. 448

Kau, B.; Rieger, M.; Sauer, H.

Modulation der biochemischen Wirkung von 5-Fluorouracil (5-FU) durch Leucovorin (LCV) gemessen anhand der Thymidylat-Synthase-Aktivität und des Nucleosid-Einbaus

in die DNS 453

Kurzmitteilungen

Krammer, B.

Ein Schnelltest-System für die Wirkung von in-vitro-Parametern

auf die Sensibilisierungs-Effizienz in der Photodynamischen

Laser-(tumor)therapie 462

Kasuistiken

Braumann, D.; Mainz,er, K.; Günzl, C; Lewerenz, B. Myokardinfarkte im Rahmen einer 5-

Fluorouracil-Therapie . . . 465

Informationen für die Klinik

Porzsolt, F.

Therapie des metastasierenden Mammakarzinoms am

Tumorzentrum Ulm 1980 und 1990468

Mitteilungen onkologischer Gesellschaften

AIO-Mitteilungen 473

Sonderbände $\quad 414$

Autorenverzeichnis 1990

478

Jahres-Inhalt 1990 
Stichwortverzeichnis 483 